\title{
O cuidado primário para as crianças com autismo na saúde brasileira
}

\author{
Primary care for children with autism in brazilian health \\ Atención primaria para niños con autismo en salud brasileña
}

\begin{abstract}
RESUMO
Objetivo: descrever os cuidados oferecidos pela atenção primária à saúde para crianças autistas no Brasil. Método: Este estudo possui caráter descritivo, qualitativo, sendo uma revisão integrativa. Foi realizado no mês de julho de 2021, nas plataformas eletrônicas: Scientific Electronic Library Online (SciELO) e Google acadêmico. Foi realizado com pesquisas entre os anos de 2017 e 2021, seguindo critérios de inclusão e exclusão. Resultados: Fizeram parte do estudo 07 publicações científicas. Os estudos trouxeram muitos desafios enfrentados pelas crianças autistas e suas famílias, e estes desafios, perpassam desde o funcionamento e integração da rede de atenção à saúde, até a capacitação dos profissionais de saúde para cuidar dessas crianças. Conclusão: Dessa forma, o cuidado primário encontra-se um pouco fragilizado no que tange a criança autista, tanto na detecção precoce do diagnóstico, quanto no acompanhamento após o diagnóstico.
\end{abstract}

DESCRITORES: Autismo; Cuidado; Atenção Primária à Saúde; Crianças

\section{ABSTRACT}

Objective: describe the care provided by primary health care for autistic children in Brazil. Method: This study is descriptive, qualitative, and an integrative review. It was carried out in July 2021, on the following electronic platforms: Scientific Electronic Library Online (SciELO) and Academic Google. It was carried out with research between 2017 and 2021, following inclusion and exclusion criteria. Results: The study included 07 scientific publications. The studies brought many challenges faced by autistic children and their families, and these challenges range from the functioning and integration of the health care network, to the training of health professionals to take care of these children. Conclusion: In this way, primary care is somewhat weakened with regard to the autistic child, both in the early detection of the diagnosis and in the follow-up after the diagnosis.

DESCRIPTORS: Autism; Caution; Primary Health Care; Kids.

\section{RESUMEN}

Objetivo: describir la atención brindada por la atención primaria de salud a los niños autistas en Brasil. Método: Este estudio es descriptivo, cualitativo e integrador de revisión. Se llevó a cabo en julio de 2021, en las siguientes plataformas electrónicas: Scientific Electronic Library Online (SciELO) y Academic Google. Se realizó con investigación entre 2017 y 2021, siguiendo criterios de inclusión y exclusión. Resultados: El estudio incluyó 07 publicaciones científicas. Los estudios trajeron muchos desafíos que enfrentan los niños autistas y sus familias, y estos desafíos van desde el funcionamiento e integración de la red de atención de salud, hasta la formación de profesionales de la salud para cuidar de estos niños. Conclusión: De esta forma, la atención primaria se encuentra algo debilitada con respecto al niño autista, tanto en la detección precoz del diagnóstico como en el seguimiento posterior al diagnóstico.

DESCRIPTORES: Autismo; Precaución; Primeros auxilios; Niños.

RECEBIDO EM: 17/05/2021 APROVADO EM: 30/08/2021

\section{IZABELITA FÉLIX DE OLIVEIRA}

Enfermeira. UNIFIP.

ORCID:0000-0001-5883 


\section{ROGÉRIA GOMES DA SILVA}

Enfermeira. Pós-graduada em Gestão em Saúde, Gestão da Clínica nas regiões de Saúde e Docência do Ensino Superior. Chefe do Núcleo de Atenção Ambulatorial/SES/PB.

ORCID: 0000-0001-7075-1369

\section{ERLÂNIA SOUZA COSTA}

Enfermeira. Pós-graduada em obstetrícia e neonatologia. Faculdade de Enfermagem Nova Esperança. ORCID: 0000-0002-5018-0404

\section{LÚCIA GOMES DE SOUZA SILVA}

Enfermeira. Faculdade Maurício de Nassau. Pós-graduada em Urgência/Emergência e Unidade de Terapia Intensiva. ORCID: 0000-0001-6998-435X

\section{KÁTIA CRISTINA BARBOSA FERREIRA}

Enfermeira. Universidade Estadual da Paraíba. ORCID: 0000-0002-3353-2973.

\section{LUCIENE DE SOUZA SANTOS ALBUQUERQUE}

Enfermeira. Centro Universitário de João Pessoa.

ORCID: 0000-0001-5119-195X.

\section{INTRODUÇÃO}

0 Transtorno do Espectro do Autismo (TEA) é um transtorno que acomete o desenvolvimento humano, geralmente identificado na infância e caracterizado por dificuldades de comunicação e interação social e pela presença de comportamentos e/ou interesses repetitivos ou restritos $^{1}$. O autismo sempre existiu, porém, só foi identificado na década de 40, pelos médicos Leo Kanner e Hans Asperger. Mediante outras pesquisas, que foram sendo realizadas, o conhecimento sobre o autismo foi disseminando para a população ${ }^{2}$.

Continuamente, com os avanços na área da saúde, as características das pessoas com autismo passaram a ser mais identificadas e percebeu-se, que os pacientes apresentam: isolamento social, movimentos repetitivos, fixações direcionadas e por vezes intensas, desvio do olhar, mudanças na linguagem, boas relações com objetivos, brinquedos, ansiedade para realizar tarefas e outras características de acordo com a gravidade da patologia ${ }^{2}$.

No Brasil, o direto à saúde é garantido para todos, conforme a Constituição Federal de 1988, que aborda no artigo 196, que a saúde é um dever do Estado, que é para todos, mediante as políticas de saúde, com o objetivo de reduzir agravos, promover, proteger e recuperar a saúde das pessoas ${ }^{3}$.

Considerando este contexto, as pessoas com autismo, possuem o direito à vida, proteção contra qualquer forma de abuso, exploração ou de origem semelhantes, direito à saúde, acesso aos serviços de saúde de acordo com as suas necessidades, alcançando: atendimento multiprofissional, diagnóstico, medicamentos, acompanhamento do desenvolvimento e outras demandas relacionadas à saúde ${ }^{4}$.

Perante a Portaria MS/GM no 4.279, de 30 de dezembro de $2010^{5}$., e o Decreto presidencial $\mathrm{n}^{\circ} 7.508$, de 28 de junho de $2011^{6}$, tem-se a Redes de Atenção à Saúde (RAS), que tem por objetivo, organizar as ações do Sistema Único de Saúde, garantir a integração entre os serviços, ofertando saúde. Dentro de uma perspectiva macro da RAS, tem-se a Rede de Atenção Psicossocial (RAPS) ${ }^{7}$ no qual a pessoa com autismo tem suporte na atenção à saúde, juntamente com os outros serviços da rede. A realidade ainda demonstra, que os profissionais de saúde possuem dificuldades para manejar as crianças que tem sinais de autismo, e por vezes, não sabem como se comunicar, ou para onde direcionar o atendimento, como proceder no diagnóstico, ou pelo menos em uma orientação, por isso, este estudo é relevante. Sendo assim, construiu-se a questão de pesquisa: Como se dá o cuidado primário para as crianças autistas no Brasil? Tem-se por objetivo: descrever os cuidados oferecidos pela atenção primária à saúde para crianças autistas no Brasil.

\section{MÉTODO}

Este estudo possui caráter descritivo, qualitativo, sendo uma revisão integrativa. Foi realizado no mês de julho de 2021, nas plataformas eletrônicas: Scientific Electronic Library Online (SciELO) e Google acadêmico. Para selecionar as publicações, seguiu-se os critérios de inclusão: publicação em inglês, espanhol e português, completos, disponíveis de forma gratuita, entre os anos de 2017 a 2021, que não apareçam em duplicidade na busca, além de responderem ao objetivo do da pesquisa. Foram excluídos: publicações fora dos critérios de inclusão.

Os descritores para a busca das publicações foram: Autismo; Cuidado; Atenção Primária à Saúde; Crianças. Estes descritores foram cruzados pelo operador booleano AND. Além disso, fez-se o uso de um instrumento simples, construído pelos autores deste estudo, para organizar as informações de cada publicação em formato de fichamento. As informações recolhidas foram: nome dos autores, título, ano de publicação, periódico e os principais achados sobre o cuidado primário para as crianças com 
Oliveira, I. F., Silva, R. G., Costa, E. S., Silva, L. G. S., Ferreira, K. C. B., Albuquerque, L. S. S.

O cuidado primário para as crianças com autismo na saúde brasileira

QUADRO 1- Apresentação das publicações sobre o cuidado primário para crianças autistas

\begin{tabular}{|c|c|c|c|c|}
\hline AUTORES & TÍTULO & $\begin{array}{l}\text { ANO DE } \\
\text { PUBLICAÇÃO }\end{array}$ & PERIÓDICO & $\begin{array}{c}\text { PRINCIPAIS ACHADOS SOBRE O CUIDADO } \\
\text { PRIMÁRIA PARA AS CRIANÇAS COM } \\
\text { AUTISMO }\end{array}$ \\
\hline $\begin{array}{l}\text { Silva Ribeiro } \\
\text { SG, Araújo } \\
\text { Ferreira Rocha } \\
\text { J, Cerazi Sartori } \\
\text { M, Valadão FB, } \\
\text { Jurado SR. (8) }\end{array}$ & $\begin{array}{l}\text { Desafios da assistência aos } \\
\text { indivíduos com transtorno do } \\
\text { espectro autista na assistência básica } \\
\text { à saúde: uma revisão de literatura }\end{array}$ & 2020 & $\begin{array}{l}\text { Revista } \\
\text { Multidisciplinar } \\
\text { em saúde. }\end{array}$ & $\begin{array}{l}\text { Dificuldade para identificar o autismo. } \\
\text { Ausência de capacitação dos profissionais de } \\
\text { saúde. } \\
\text { Pouca tecnologia disponível para auxiliar no } \\
\text { cuidado que é oferecido e na comunicação. }\end{array}$ \\
\hline $\begin{array}{c}\text { Costa CS, } \\
\text { Guarany NR. (9) }\end{array}$ & $\begin{array}{c}\text { O reconhecimento dos sinais de } \\
\text { autismo por profissionais atuantes } \\
\text { nos serviços de puericultura na } \\
\text { atenção básica. }\end{array}$ & 2021 & $\begin{array}{l}\text { Interinstitutional } \\
\text { Brazilian Journal } \\
\text { of Occupational } \\
\text { Therapy. }\end{array}$ & $\begin{array}{l}\text { Nenhum profissional possui formação sobre } \\
\text { autismo. } \\
\text { Os profissionais de saúde têm interesse em } \\
\text { se capacitar no tema. } \\
\text { Possuem dificuldades na identificação dos } \\
\text { sintomas no processo de desenvolvimento } \\
\text { infantil. }\end{array}$ \\
\hline $\begin{array}{l}\text { Bortone ART, } \\
\text { Wingester ELC. } \\
\text { (10) }\end{array}$ & $\begin{array}{l}\text { Identificação do espectro do } \\
\text { transtorno autista durante o } \\
\text { crescimento e o desenvolvimento } \\
\text { infantil: o papel do profissional de } \\
\text { enfermagem. }\end{array}$ & 2016 & $\begin{array}{l}\text { Revista Digital } \\
\text { FAPAM. }\end{array}$ & $\begin{array}{l}\text { Poucos estudos sobre a enfermagem na } \\
\text { identificação dos sinais de autismo. } \\
\text { Necessário maiores estímulos entre os } \\
\text { profissionais de saúde. }\end{array}$ \\
\hline $\begin{array}{l}\text { Rezende LO de. } \\
\text { et al. (11) }\end{array}$ & $\begin{array}{l}\text { Conhecimento sobre Transtorno do } \\
\text { Espectro Autista entre profissionais } \\
\text { da atenção básica de saúde. }\end{array}$ & 2020 & $\begin{array}{l}\text { Manuscripta } \\
\text { Médica. }\end{array}$ & $\begin{array}{c}\text { Os achados mostraram que os médicos } \\
\text { apresentaram um melhor conhecimento no } \\
\text { que diz respeito ao TEA. } \\
\text { Evidenciou-se a necessidade educação } \\
\text { continuada para capacitar os profissionais } \\
\text { que acompanham as crianças. }\end{array}$ \\
\hline $\begin{array}{l}\text { David Amaral, } \\
\text { L., Fabiano de } \\
\text { Carvalho, T. y } \\
\text { Barreto Bezerra, } \\
\text { A. C. (12) }\end{array}$ & $\begin{array}{l}\text { Atenção bioética à vulnerabilidade } \\
\text { dos autistas: A odontologia na } \\
\text { estratégia da saúde da família. }\end{array}$ & 2016 & $\begin{array}{l}\text { Revista } \\
\text { Latinoamericana } \\
\text { de Bioética. }\end{array}$ & $\begin{array}{c}\text { Sugere-se um protocolo de atenção } \\
\text { odontológica ao autista. } \\
\text { O tratamento odontológico do paciente } \\
\text { autista deve ter curta duração e de forma } \\
\text { organizada. A comunicação com o paciente } \\
\text { deve ser feita através de comandos claros } \\
\text { e objetivos, com reforços positivos ou } \\
\text { negativos. }\end{array}$ \\
\hline $\begin{array}{l}\text { Martins RA. et } \\
\text { al. (13) }\end{array}$ & $\begin{array}{l}\text { Assistência do enfermeiro à criança } \\
\text { autista na atenção básica. }\end{array}$ & 2021. & $\begin{array}{l}\text { Brazilian Journal } \\
\text { of Health Review. }\end{array}$ & $\begin{array}{l}\text { Fragilidade de Sistema Único de Saúde (SUS) } \\
\text { no atendimento as pessoas com Transtorno } \\
\text { do Espectro Autista. } \\
\text { Ausência de conhecimentos teóricos sobre as } \\
\text { especificidades do autismo. }\end{array}$ \\
\hline
\end{tabular}




\begin{tabular}{|c|c|c|c|c|}
\hline $\begin{array}{c}\text { Ranalli, NMG. } \\
(14)\end{array}$ & $\begin{array}{c}\text { Impacto de um modelo de } \\
\text { capacitação sobre sinais precoces } \\
\text { de autismo para profissionais da } \\
\text { atenção básica de saúde. }\end{array}$ & 2017. & $\begin{array}{c}\text { Universidade } \\
\text { Presbiteriana } \\
\text { Mackenzie. }\end{array}$ & $\begin{array}{c}\text { Sensibilidade quanto ao conhecimento sobre } \\
\text { o autismo e seus sinais de alerta. }\end{array}$ \\
\hline
\end{tabular}

Fonte: Elaborado pelo autor,2020.

autismo no Brasil.

$\mathrm{Na}$ busca das publicações, foram encontradas 145 publicações, e após o filtro de período de publicação, somente 100 seguiram na análise. Foi realizada a leitura dos títulos, no qual 25 publicações direcionavam para o tema escolhido. Após a leitura dos resumos das 25 publicações, apenas 07 estavam dentro do objetivo principal da pesquisa. Foi realizada a leitura integral das 07 publicações, e todas permaneceram na pesquisa. Houve a validação da busca das publicações, realizada por dois pesquisadores da temática.

\section{RESULTADOS}

Dessa forma, o corpo de resultados foi composto por 07 publicações sobre o tema. Ocorreu o processo de validação da busca por dois pesquisadores da área. Assim, as publicações foram expostas em quadro único, com o auxílio do programa da Microsoft Word.

Obteve-se duas publicações nos anos de 2016, 2020 e 2021 e apenas uma no ano de 2017. Todos os estudos trouxeram muitos desafios enfrentados pelas crianças autistas e suas famílias, e estes desafios, perpassam desde o funcionamento e integração $\mathrm{da}$ rede de atenção à saúde, até a capacitação dos profissionais de saúde para cuidar dessas crianças. Dessa forma, o cuidado primário encontra-se um pouco fragilizado no que tange a criança autista, tanto na detecção precoce do diagnóstico, quanto no acompanhamento após o diagnóstico.

\section{DISCUSSÃO}

Diante dos resultados encontrados, compreende-se, que o Brasil vem trazendo novas estratégias, com outros modelos de atenção à saúde, como por exemplo da rede de atenção à saúde mental, bem como,

\section{O cuidado}

primário precisa

ser humanizado,

com paciência,

dedicação,

singularidade e

amor, diante de

situações que

necessitam de maior

atenção

a rede para pessoas com deficiência. A reestruturação do modelo de saúde foi um passo importante para organizar a assistência que estava sendo oferecida, neste caso, para as crianças com autismo ${ }^{13-15}$. Sabe-se que a assistência que é desempenhada para a criança, precisa atender as suas necessidades, abrangendo também a família ${ }^{13-16}$.

O cuidado primário precisa ser humanizado, com paciência, dedicação, singularidade e amor, diante de situações que necessitam de maior atenção ${ }^{13-17}$. A literatura aponta, que a criança com autismo é espontânea, diz aquilo que pensa, e por isso, é importante tornar o cuidado algo lúdico, sem que seja imposto para ela, gerando ansiedade na criança. A comunicação também é importante em qualquer etapa do cuidado primário, usar recursos como a musicoterapia, teatro, gestos, brincadeiras e desenhos, podem ajudar a reduzir o medo da criança, tornando-a mais próxima do profissional de saúde que está lhe acolhendo ${ }^{16}$.

$\mathrm{Na}$ atenção básica, o profissional de saúde precisa estar capacitado, e encontrar os sinais de autismo desde a fase inicial, agindo precocemente $e^{13-16-18}$. Os estudos que participaram da pesquisa, identificaram um déficit neste ponto, no qual os profissionais de saúde, não possuíam o conhecimento devido para avaliar os sinais de autismo, e ainda, não tinham o direcionamento das melhores condutas após a identificação dos sinais de alerta $^{8-10-11-13}$.

O cuidado primário é multiprofissional, foi visto, que o enfermeiro está bem próximo das crianças, por realizar a puericultura e acompanhar o desenvolvimento infantil, junto com os demais profissionais da equi$\mathrm{pe}^{12}$. Porém, também ficou evidenciado, que a falta de capacitação é uma problemática, pois não há investimentos da gestão em saúde para isso. Na saúde bucal, o tratamento o odontológico também precisa ser objetivo, humanizado, com acolhimento, suporte familiar, e se possível, com agendamento, visando o bem-estar da criança ${ }^{12}$.

Rezende aponta ${ }^{11}$, que as crianças com autismo também ficam doentes de outras patologias, e tem momento agudos, precisando de outros serviços de saúde. E a falta de conhecimento dos profissionais, na forma de abordagem, comunicação e até nas condutas, dificulta o processo de atenção à saúde dentro da RAS.

Bortone e colaboradores ${ }^{10}$, ressaltam, que existe uma escassez de publicações sobre o tema, e quando se trata da enfermagem e da avaliação profissional para crianças com autismo, os estudos continuam insuficientes. Os profissionais de saúde precisam de embasamento teórico para melhorarem a sua prática, e as publicações oferecem isso, portanto, faz-se necessário o incentivo de 


\section{artigo}

Oliveira, I. F., Silva, R. G., Costa, E. S., Silva, L. G. S., Ferreira, K. C. B., Albuquerque, L. S. S

O cuidado primário para as crianças com autismo na saúde brasileira

produção e publicação de materiais. Costa e os demais autores ${ }^{9}$, compreendem que a assistência em saúde precisa visar a promoção do cuidado, e a atenção básica é fundamental nesse processo, cuidando individualmente e coletivamente, desde a prevenção até a reabilitação.

\section{CONCLUSÃO}

A pesquisa alcançou o seu objetivo, tra- zendo questóes importantes sobre um tema primordial para a saúde coletiva. Compreendeu-se a necessidade de novas pesquisas nesta área, pois os resultados descrevem a dificuldade para oferecer suporte teórico aos profissionais e melhorar o atendimento para as crianças.

É preciso o incentivo das gestões estaduais e municipais para avançar nos cuidados primários que são oferecidos as crianças com autismo. A capacitação dos profissio- nais é essencial, visando a identificação precoce, monitoramento e acompanhamento do desenvolvimento infantil de forma saudável. A RAS precisa estar integrada, de forma ampla, intersetorial, multidisciplinar e efetiva, para garantir o acesso à saúde. Por fim, entende-se que todos os profissionais trabalham de forma unificada no cuidado à criança com autismo, e que a família precisa estar inserida neste contexto, objetivando uma assistência à saúde inclusiva.

\section{REFERÊNCIAS}

1. Araújo de LA. et al. Transtorno do Espectro do Autismo. Sociedade Brasileira de Pediatria, 2019.

2. Brasil. Linha de Cuidado para a Atenção às Pessoas com transtornos do Espectro do Autismo e suas famílias na Rede De Atenção Psicossocial Do Sistema Único De Saúde. 2015.

3. Brasil. Constituição Federal Brasileira. 1988.

4. Brasil. Estratégias para o cuidado da pessoa com doença crônica. 2015.

5. Brasil. Portaria N 4.279, DE 30 DE Dezembro DE 2010. 2010

.6. Brasil. Decreto n 7.508, de 28 de junho de 2011. 2011.

7. Brasil. Redes de Atenção à Saúde. 2014.

8. Silva Ribeiro SG, Araújo Ferreira Rocha J, Cerazi Sartori M, Valadão FB, Jurado SR. Desafios da assistência aos indivíduos com transtorno do espectro autista na assistência básica à saúde: uma revisão de literatura. Revista Multidisciplinar em saúde.v.1, n.2, 2020.

9. Costa CS, Guarany NR. O reconhecimento dos sinais de autismo por profissionais atuantes nos serviços de puericultura na atenção básica. Interinstitutional Brazilian Journal of Occupational Therapy. v.5, n.1, 2021.

10. Bortone ART, Wingester ELC. Identificação do espectro do transtorno autista durante o crescimento e o desenvolvimento infantil: o papel do profissional de enfermagem. Revista Digital FAPAM. v.7, n7, 2016

11. Rezende LO de. et al. Conhecimento sobre Transtorno do Espectro Autista entre profissionais da atenção básica de saúde.
Manuscripta Médica. 3:31-39, 2021.

12. David Amaral, L., Fabiano de Carvalho, T. y Barreto Bezerra, A. C. Atenção bioética à vulnerabilidade dos autistas: A odontologia na estratégia da saúde da família. Revista Latinoamericana de Bioética. V.16, n.1, 2016.

13. Martins RA. et al. Assistência do enfermeiro à criança autista na atenção básica. Brazilian Journal of Health Review. v.4, n.3, 2021.

14. Ranalli, NMG. Impacto de um modelo de capacitação sobre sinais precoces de autismo para profissionais da atenção básica de saúde. Universidade Presbiteriana Mackenzie. Dissertação de Mestrado. 2017.

15. Silva LS; Furtado LAR. O sujeito autista na Rede SUS: (im) possibilidade de cuidado. Fractal: Revista de Psicologia, v. 31, n. 2, maio-ago. 2019, p. 119-129.

16. Franzoi MAH et al. Intervenção musical como estratégia de cuidado de enfermagem a crianças com transtorno do espectro do autismo em um Centro de Atenção Psicossocial. Texto Contexto Enferm, 2016; 25(1):e1020015, p. 01-08.

17. Oliveira BDC et al. Políticas para o autismo no Brasil: entre a atenção psicossocial e a reabilitação. Physis Revista de Saúde Coletiva, Rio de Janeiro, 27 [ 3 ]: 2017, p.707-726.

18. Fernandes ADSA. et al. Desafios cotidianos e possibilidades de cuidado às crianças e adolescentes com Transtorno do Espectro Autista (TEA) frente à COVID-19. Cadernos Brasileiros de Terapia Ocupacional/Brazilian Journal of Occupational Therapy, Preprint, 2020, p. 01-17. 